\title{
Mutant prevention concentration of tigecycline for carbapenem-susceptible and -resistant Acinetobacter baumannii
}

\author{
Jun-Chang Cui, You-Ning Liu and Liang-An Chen
}

The Journal of Antibiotics (2010) 63, 29-31; doi:10.1038/ja.2009.111; published online 13 November 2009

Keywords: Acinetobacter baumannii; mutant prevention concentration; propensity for selection of resistance; resistance; tigecycline

Acinetobacter baumannii has become a serious nosocomial pathogen, particularly with cases of ventilator-associated pneumonia and bacteraemia. ${ }^{1}$ In recent years, many outbreaks of nosocomial infections caused by carbapenem-resistant $A$. baumannii have been reported. ${ }^{2,3}$ These carbapenem-resistant isolates are often resistant to multiple agents, such as cephalosporins, fluoroquinolones, aminoglycosides, tetracyclines and sulbactam. ${ }^{2,3}$ Carbapenems once had a crucial role in the management of serious nosocomial A. baumannii infections. Unfortunately, the prevalence of carbapenem-resistant isolates appears to be increasing. In China, the rate of carbapenem-resistance in A. baumannii isolates was $40-60 \%$ in $2005 .{ }^{4}$ These features make A. baumannii infections very difficult to treat as options are limited. A new broad-spectrum glycylcycline, tigecycline, shows good in vitro activity against $A$. baumanni $i^{5,6}$ and is now considered a good treatment option for multidrug-resistant $A$. baumannii infections. ${ }^{7,8}$ However, tigecycline-resistant A. baumannii has been observed after tigecycline treatment of $A$. baumannii infections. ${ }^{7-9}$ Whether tigecycline is particularly prone to emergence of resistance with A. baumannii is unknown.

Mutant prevention concentration (MPC) and the mutant selection window theory has been proposed as new measures for propensity of an antimicrobial to develop resistance. ${ }^{10}$ To assess the propensity for A. baumannii to develop resistance, we measured the MPC of tigecycline. Eighty A. baumannii sputum isolates were collected from three hospitals in Beijing. Fifty were carbapenem-resistant isolates ( $\mathrm{MIC} \geqslant 16 \mu \mathrm{g} \mathrm{ml}^{-1}$ to either meropenem or imipenem); they were also resistant to broad-spectrum beta-lactams, aminoglycosides and fluoroquinolones. Thirty isolates were susceptible to meropenem, imipenem, aminoglycosides and fluoroquinolones. Staphylococcus aureus ATCC 29213 and Escherichia coli ATCC 25922 served as quality-control strains. Bacterial cultures were grown in MullerHinton broth or on Muller-Hinton agar at $37^{\circ} \mathrm{C}$. Stock solutions of tigecycline ( $5.12 \mathrm{mg} \mathrm{ml}^{-1}$ ) were prepared from i.v. injection powder (Wyeth Pharmaceutical, Philadelphia, PA, USA) in sterile water. MIC was determined according to Clinical and Laboratory Standards Institute using Muller-Hinton agar. As no interpretive criterion for tigecycline with $A$. baumannii has been established, Clinical and Laboratory Standards Institute interpretive criteria for Enterobacteriaceae were used for A. baumannii, as had been reported in previous literature (susceptible MIC $\leqslant 2 \mu \mathrm{g} \mathrm{m}^{-1}$; intermediate MIC, $>2$ but $<8 \mu \mathrm{g} \mathrm{ml}^{-1}$; resistant MIC, $\left.\geqslant 8 \mu \mathrm{g} \mathrm{ml}^{-1}\right){ }^{7,8}$ For determination of the MPC, high-density cultures were prepared from overnight cultures grown in liquid medium followed by a 10 -fold dilution and $4 \mathrm{~h}$ of incubation with shaking at $37^{\circ} \mathrm{C}$. Then, the suspension was centrifuged $(4000 \mathrm{~g}$ for $10 \mathrm{~min}$ ) and re-suspended in fresh Mueller-Hinton broth to yield a concentration of about $10^{10}$ c.f.u. ml ${ }^{-1}$. A series of agar plates containing known antibiotic concentrations were then inoculated with $\sim 10^{10}$ c.f.u. of $A$. baumannii. The inoculated plates were incubated for $72 \mathrm{~h}$ at $37^{\circ} \mathrm{C}$ and screened visually for growth. MPC was defined as the lowest drug concentration preventing recovery of colonies when $>10^{10}$ cells were tested. ${ }^{11}$ Rectilinear correlation and regression were used with STATA 7.0 software (StataCorp LP, College Station, TX, USA) for correlating MIC and MPC.

With carbapenem-resistant isolates, $\mathrm{MIC}_{90}$ and $\mathrm{MIC}_{\text {range }}$ were 2 and $0.25-2 \mu \mathrm{g} \mathrm{ml}^{-1}$, respectively. $\mathrm{MPC}_{90}$ and $\mathrm{MPC}_{\text {range }}$ were 64 and $4-64 \mu \mathrm{g} \mathrm{ml}^{-1}$, respectively. For carbapenem-susceptible clinical isolates, $\mathrm{MIC}_{90}$ and $\mathrm{MIC}_{\text {range }}$ were 1 and $0.125-2 \mu \mathrm{g} \mathrm{ml}^{-1}$; $\mathrm{MPC}_{90}$ and $\mathrm{MPC}_{\text {range }}$ were 16 and $1-32 \mu \mathrm{g} \mathrm{ml}^{-1}$, respectively. Using the susceptibility breakpoint of $\leqslant 2 \mu \mathrm{g} \mathrm{ml}^{-1}$, all 80 isolates were susceptible to tigecycline. However, MPC with all 50 carbapenem-resistant isolates was 2- to 32-fold higher than the susceptibility breakpoint. Only 10 of 30 carbapenem-susceptible isolates had MPC values $\leqslant 2 \mu \mathrm{g} \mathrm{ml}^{-1}$ susceptibility breakpoint. Carbapenem-resistant isolates had MPC values 2- to 4-fold higher than carbapenem-susceptible 

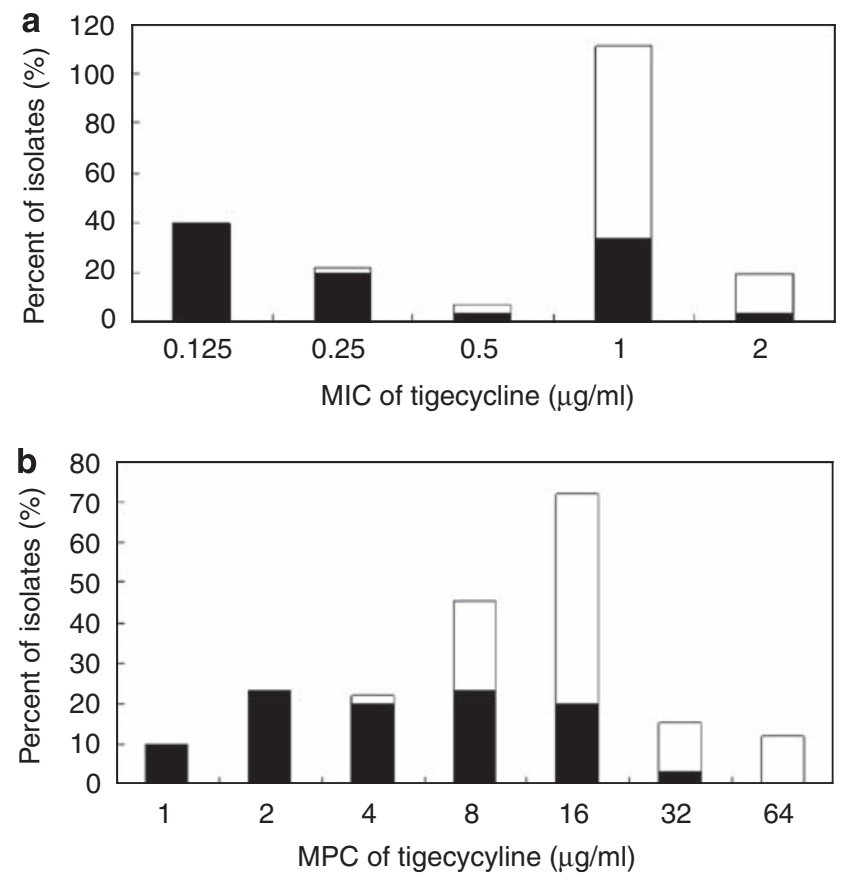

Figure 1 Distribution of minimum inhibitory concentration (a) and mutant prevention concentration (b) for clinical isolates of $A$. baumannii. Solid bars indicate carbapenem-susceptible isolates $(n=30)$, open bars represent carbapenem-resistant isolates $(n=50)$.

isolates. The MICs of tigecycline for carbapenem-resistant isolates tended to be higher than those for carbapenem-susceptible isolates (Figure 1). Consistent with a previous report, ${ }^{12}$ poor correlation between MPC and MIC was observed with both carbapenem-resistant $\left(r^{2}=0.60\right)$ and carbapenem-susceptible isolates $\left(r^{2}=0.77\right)$; thus MIC cannot be used to reliably predict MPC.

MPC and the mutant selection window theory has suggested that mutant enrichment occurs when the concentration of the antimicrobial agent is high enough to block the growth of susceptible cells (above the MIC) but not that of resistant mutants (below the MPC). Keeping antimicrobial agent concentrations at the site of infection above the MPC is expected to restrict selective enrichment. ${ }^{10}$ So MPC can be linked with antimicrobial pharmacokinetics to assess the propensity of the agent to develop resistance. Although there is a debate regarding the utility of MPC concept beyond fluoroquinolones, ${ }^{13} \mathrm{Zhao}^{14}$ thinks MPC should apply to a broad range of genetic resistance problems, not just those caused by fluoroquinolones. The MPC concept is relatively insensitive to the first step in the development of resistance, individual resistance mechanisms. It addresses the second step, the selective enrichment of mutant subpopulations. This makes the MPC approach likely to apply to many classes of antibiotics, including tigecycline. Pharmacokinetic measurements with tigecycline indicate that the maximum concentrations in serum and lung epithelial lining fluid of healthy subjects $(N=30)$ were 0.72 and $0.37 \mu \mathrm{g} \mathrm{ml}^{-1}$, respectively, when tigecycline was administered as a $100 \mathrm{mg}$ first dose followed by six $50 \mathrm{mg}$ doses given every $12 \mathrm{~h} .^{15}$ Sun et al. ${ }^{16}$ reported that the maximum concentrations of tigecycline in serum and skin blister fluid of healthy subjects $(N=10)$ were 0.82 and $0.27 \mu \mathrm{g} \mathrm{ml}^{-1}$, respectively. Although MIC for tigecycline with A. baumannii is below the susceptibility breakpoint $\left(\leqslant 2 \mu \mathrm{g} \mathrm{ml}^{-1}\right)$, the MPC is above the attainable surum, lung tissue and skin tissue levels. For carbapenem-resistant A. baumannii clinical isolates, $\mathrm{MPC}_{90}$ of tigecycline was $64 \mu \mathrm{g} \mathrm{ml}^{-1}$ and it is above the serum, lung epithelial lining fluid and skin blister fluid concentrations significantly. For carbapenem-susceptible A. baumannii clinical isolates, $\mathrm{MPC}_{90}$ of tigecycline was $16 \mu \mathrm{g} \mathrm{ml}^{-1}$. Though $\mathrm{MPC}_{90}$ of susceptible isolates was significantly lower than that of carbapenem-resistant isolates, it was still higher than the tigecycline concentrations in serum, lung epithelial lining fluid and skin blister fluid. As the concentrations of tigecycline in epithelial lining fluid of infected murine lungs were higher than those of uninfected lungs, ${ }^{17}$ it is anticipated that the concentrations of tigecycline in epithelial lining fluid of infected patients would be higher than those of healthy subjects. But the concentrations of tigecycline in epithelial lining fluid of healthy subjects were significantly lower than the $\mathrm{MPC}_{90}$, and it is also anticipated that the concentrations in epithelial lining fluid of infected patients would be lower than the MPC $_{90}$. Thus, tigecycline concentrations would fall between the MIC and the MPC for most time of the entire dosing period when this compound was used to treat A. baumannii infections. As mutant enrichment readily occurs when antimicrobial concentrations fall between the MIC and the MPC, ${ }^{10,18}$ treatment of A. baumannii with tigecycline is expected to selectively enrich resistant mutant subpopulations. Such prediction is consistent with recent reports of emergence of tigecycline-resistant $A$. baumannii after tigecycline therapy. ${ }^{7-9}$ Although decreased susceptibility to tigecycline in clinical isolates of $A$. baumannii is associated with the overexpression of the AdeABC multidrug efflux pump, ${ }^{19}$ the proposed mechanism of resistant isolates selected in vitro need further study. Our data suggest that tigecycline may be prone to emergence of resistance with A. Baumannii; thus, close monitoring of A. Baunmanii susceptibilities and patient responsiveness to tigecycline monotherapy is warranted.

\section{ACKNOWLEDGEMENTS}

We thank Dr Xilin Zhao for critical comments on the paper. This work was supported by grants from National Natural Science Foundation of China (CNSFC Grants 30672505).

1 Cisneros, J. M. et al. Bacteremia due to Acinetobacter baumannii: epidemiology, clinical findings, and prognostic features. Clin. Infect. Dis. 22, 1026-1032 (1996).

2 Valencia, R. et al. Nosocomial outbreak of infection with pan-drug-resistant Acinetobacter baumannii in a tertiary care university hospital. Infect. Control Hosp. Epidemiol. 30, 257-263 (2009).

3 Zong, Z., Lü, X., Valenzuela, J. K., Partridge, S. R. \& Iredell, J. An outbreak of carbapenem-resistant Acinetobacter baumannii producing OXA-23 carbapenemase in western China. Int. J. Antimicrob. Agents. 31, 50-54 (2008).

4 Wang, Y. \& Xu, Y. CHINET surveillance of antimicrobial resistance among $A$. baumannii isolates in China during 2004-2005. Chin. J. Infect. Chemother. 7, 279-282 (2007).

5 Hoban, D. J., Bouchillon, S. K. \& Dowzicky, M. J. Antimicrobial susceptibility of extendedspectrum beta-lactamase producers and multidrug-resistant Acinetobacter baumannii throughout the United States and comparative in vitro activity of tigecycline, a new glycylcycline antimicrobial. Diagn. Microbiol. Infect. Dis. 57, 423-428 (2007).

6 Souli, M. et al. In vitro activity of tigecycline against multiple-drug-resistant, including pan-resistant, Gram-negative and Gram-positive clinical isolates from Greek hospitals. Antimicrob. Agents Chemother. 50, 3166-3169 (2006).

7 Anthony, K. B. et al. Clinical and microbiological outcomes of serious infections with multidrug-resistant Gram-negative organisms treated with tigecycline. Clin. Infect. Dis. 46, 567-570 (2008)

8 Schafer, J. J., Goff, D. A., Stevenson, K. B. \& Mangino, J. E. Early experience with tigecycline for ventilator-associated pneumonia and bacteremia caused by multidrugresistant Acinetobacter baumannii. Pharmacotherapy 27, 980-987 (2007).

9 Reid, G. E., Grim, S. A., Aldeza, C. A., Janda, W. M. \& Clark, N. M. Rapid development of Acinetobacter baumannii resistance to tigecycline. Pharmacotherapy 27, 1198-1201 (2007).

10 Zhao, X. \& Drlica, K. Restricting the selection of antibiotic-resistant mutants: a general strategy derived from fluoroquinolone studies. Clin. Infect. Dis. 33 (Suppl. 3), S147-S156 (2001).

11 Blondeau, J. M., Zhao, X., Hansen, G. \& Drlica, K. Mutant prevention concentration of fluoroquinolones for clinical isolates of Streptococcus pneumoniae. Antimicrob. Agents Chemother. 45, 433-438 (2001). 
12 Drlica, K., Zhao, X., Blondeau, J. \& Hesje, C. Low correlation between MIC and mutant prevention concentration. Antimicrob. Agents Chemother. 50, 403-404 (2006).

13 Smith, H. J., Nichol, K. A., Hoban, D. J. \& Zhanel, G. G. Stretching the mutant prevention concentration (MPC) beyond its limits. J. Antimicrob. Chemother. 51, 1323-1325 (2003).

14 Zhao, X. Clarification of MPC and the mutant selection window concept. J. Antimicrob. Chemother. 52, 731 (2003).

15 Conte Jr., J. E., Golden, J. A., Kelly, M. G. \& Zurlinden, E. Steady-state serum and intrapulmonary pharmacokinetics and pharmacodynamics of tigecycline. Int. J. Antimicrob. Agents 25, 523-529 (2005).
16 Sun, H. K. et al. Pharmacokinetic profile of tigecycline in serum and skin blister fluid of healthy subjects after multiple intravenous administrations. Antimicrob. Agents Chemother. 49, 1629-1632 (2005).

17 Crandon, J. L., Kim, A. \& Nicolau, D. P. Comparison of tigecycline penetration into the epithelial lining fluid of infected and uninfected murine lungs. J. Antimicrob. Chemother. 64, 837-839 (2009).

$18 \mathrm{Cui}$, J. et al. The mutant selection window in rabbits infected with Staphylococcus aureus. J. Infect. Dis. 194, 1601-1608 (2006).

19 Ruzin, A., Keeney, D. \& Bradford, P. A. AdeABC multidrug efflux pump is associated with decreased susceptibility to tigecycline in Acinetobacter calcoaceticusAcinetobacter baumannii complex. J. Antimicrob. Chemother. 59, 1001-1004 (2007). 